\title{
Non-periodic isochronous oscillations in plane differential systems
}

\author{
M. SABATINI
}

April 19, 2002

\section{Introduction}

Let us consider an autonomous differential system

$$
\dot{x}=P(x, y), \quad \dot{y}=Q(x, y),
$$

where $P(x, y)$ and $Q(x, y)$ are $C^{1}$ functions defined in an open connected subset $U$ of the real plane. We denote by $W$ the vector field $(P, Q)$.

Plane differential systems were widely studied for their relevance in applications. Several physical, chemical, biological systems can be successfully modelled by means of systems of type (1) (see, e. g., [3]). In particular, the study of second order O.D.E.'s arising from mechanics or the study of electric circuits

$$
\ddot{x}+h(x, \dot{x})=0
$$

can be reduced to that of suitable plane systems. A main problem is that of studying the existence and properties of oscillating solutions. With this purpose, different methods have been used to study isolated periodic solutions or non-isolated ones. The second case usually occurs when the system is integrable, as in the case of systems generated by conservative forces. When the system has a stable equilibrium position, as for the pendulum equations or for the Lotka-Volterra equations, this leads to consider a critical point $O$ of (1) surrounded by cycles. In general, an isolated critical point $O$ of (1) is said to be a center if it has a punctured neighbourhood filled with nontrivial cycles. The stability of $\mathrm{O}$ does not imply that of the cycles close to $\mathrm{O}$. In fact, a non-isolated cycle is Liapunov stable if and only if every neighbouring cycle has the same period. This motivates the definition of isochronicity: a center $\mathrm{O}$ is said to be isochronous if every orbit close enough to $\mathrm{O}$ has the same minimal period. Isochronicity has been widely studied not only for its physical meaning and for its role in stability theory, but also for its relationship with bifurcation and boundary value problems.

When $\mathrm{O}$ is a center, it is possible to construct a curve $\eta$ that meets all the cycles contained in a neighbourhood of $\mathrm{O}$. If $\mathrm{O}$ is isochronous, then every such a curve is crossed at equal time intervals by the solutions encircling $\mathrm{O}$. When this occurs, a curve $\eta$ is said to be an isochronous section. In several cases such a curve coincides with the line $x=0$ or $y=0$ (see [2]). If (2) models a physical system and is studied by means of the equivalent system

$$
\dot{x}=y, \quad \dot{y}=h(x, \dot{x}),
$$


this means that the position $x=0$, or the speed $y=\dot{x}=0$ are assumed at equal time intervals.

Isochronous sections may appear also in non-integrable systems. A position can be assumed at equal time intervals even in presence of friction, as in mechanical systems, or in presence of energy dissipation associated with heat production, as in electric circuits. In this case the system is characterized by the presence of oscillations with decreasing width, performed at equal time intervals. Moreover, isochronous oscillations may appear in a neighbourhood of a limit cycle $\Gamma$. If $\Gamma$ is attracting, this impies the existence of both oscillations with increasing width and oscillations with decreasing width, all performed at equal time intervals.

In this paper we study isochronous sections of non-integrable plane systems, trying both to prove the existence of such curves, and to identify them. We adapt some techniques usually applied to study centers' isochronicity.

In next section we consider isochronous sections in a neighbourhood of critical points of focus type. We first look for transformations taking (1) into a new system having, in polar coordinates, the form

$$
\dot{\rho}=f(\rho, \theta), \quad \dot{\theta}=g(\theta) \neq 0 .
$$

A simple argument shows the existence of isochronous sections. We show that under hypotheses similar to those ones considered in [10], there exists a class of Liénard equations

$$
\ddot{x}+f(x) \dot{x}+g(x)=0,
$$

with isochronous damped oscillations.

Then we consider the commutators' method, which is naturally related to the definition of isochronous section. We prove that if (1) has a nontrivial commutator $W_{1}$ with a star node at $\mathrm{O}$, then every orbit of the commutator is an isochronous section of (1). A wide collection of systems having a commutator of the required form can be constructed starting from the systems listed in [2]. Several systems have commutators with half-lines as orbits, so that isochronous sections can be easily found.

The last section is devoted to the study of systems with limit cycles. In this case isochronicity is associated to the existence of a non-costant periodic solution, which is asymptotically approximated by neighbouring solutions. The existence of an isochronous section ensures that the approximation occurs while performing oscillations of the same period, coinciding with the period of the periodic solution. Such a situation was already considered in [6] in relation to the study of mathematical models of biological systems. In [6] the existence of isochronous sections was proved for hyperbolic limit cycles. We give a sufficient condition for the existence of an isochronous section in absence of hyperbolicity. Also in this case we can give an application to the study of a class of Liénard equations, for which we find limit cycles with isochronous sections.

\section{Isochronous sections at critical points}

We start formalizing the intuitive idea of isochronicity, related to the existence of curves met at equal time intervals.

For every $z \equiv(x, y) \in \mathbb{R}^{2}$, we denote by $\psi(t, z)$ the solution of (1) such that $\psi(0, z)=z$. We denote by $-\psi$ the negative local flow defined by $(1),-\psi(t, z)=$ 
$\psi(-t, z)$. Let $(\rho, \theta)$ denote the polar coordinates of a point in the plane. We denote by $\rho(\psi(t, z))$, resp. $\theta(\psi(t, z))$, the radius and the argument of $\psi(t, z)$.

Definition 1 We say that an isolated critical point $O$ of (1) is a center if there exists a neighbourhood $U$ of $O$ such that every noncritical orbit starting at a point of $U$ is a cycle. We say that an isolated critical point $O$ of (1) is a focus if there exists a neighbourhood $U$ of $O$ containing no cycles, and such that for every noncritical orbit $\psi(t, z)$ starting at a point $z \in U$, the function $\theta(\psi(t, z))$ is increasing (decreasing) and diverging to $+\infty(-\infty)$. We say that an isolated critical point $O$ of (1) is a star node if the linear part of (1) at $O$ has equal non-zero real eigenvalues.

Definition 2 Let $O$ be an isolated critical point of (1). Let $\eta:[0,+\infty) \rightarrow \mathbb{R}^{2}$ be a $C^{1}$ curve such that $\lim _{s \rightarrow+\infty} \eta(s)=O$. Then we say that $\eta$ is an isochronous section of (1) at $O$ if either $\psi$ or $-\psi$ has the following property:

There exists $T>0$ such that $\forall z \in \eta$, one has

(i) $\psi(n T, z) \in \eta$, for every positive integer $n$;

(ii) $\psi(t, z) \notin \eta$, for $t>0, t \neq n T$.

Remark 1 If a system has a center or a focus at $O$ and an isochronous section $\eta$ in a neighbourhood of $O$, then every curve $s \mapsto \psi(t, \eta(s))$, with $0<t<T$, is an isochronous section of the system at $O$. Hence, if a system has an isochronous section, then it has infinitely many isochronous sections.

The definiton of isochronous section is based on set-theoretical properties, hence it is invariant by bijections. In next lemma we show the existence of invariant sections when the system can be transformed into a system of a special form.

From now on, we place the critical point $\mathrm{O}$ at the origin $(0,0)$. Let $U$ be a neighbourhood of $\mathrm{O}$. We say that a transformation $\Lambda: U \rightarrow \mathbb{R}^{2}$ is a local diffeomorphism at $\mathrm{O}$ if there exists a neighbourhood $U^{\prime} \subset U$ such that $\Lambda$ is a diffeomorphism between $U^{\prime}$ and $\Lambda\left(U^{\prime}\right)$.

Lemma 1 Let $O$ be a focus for (1). If there exists a local diffeomorphism $\Lambda$ at $O$ taking (1) into a system having, in polar coordinates, the form

$$
\dot{\rho}=f(\rho, \theta), \quad \dot{\theta}=g(\theta) \neq 0 .
$$

then (1) has an isochronous section at $O$.

Proof. It is sufficient to show that (5) has an isochronous section. Let us consider a ray $R_{\phi}=\{(\rho, \theta): \theta=\phi\}$. O is a focus, hence $R_{\phi}$ is crossed infinitely many times as $t$ goes to $+\infty(-\infty)$. The time passing between two consecutive intersections is

$$
T=\int_{0}^{2 \pi} \frac{d \theta}{|g(\theta)|}
$$

The value of such an integral does not depend on $\rho$, hence it is the same for all the solutions starting at a point of $R_{\phi}$ close enough to the origin.

Under the hypotheses of lemma 1, the isochronous sections of (1) are the counterimages of rays, that is the line segments $\Lambda^{-1}\left(R_{\phi}\right), \phi \in[0,2 \pi)$. Systems of the type (5) 
were considered in [4], in order to study the isochronicity of centers. Centers of such systems were called uniformly isochronous centers.

A special case of lemma 1 occurs when (1) can be linearized, that is, when there exists a local diffeomorphism at $\mathrm{O}$ taking (1) into a linear system. If the system is analytic, this is possible under rather general hypotheses, as a consequence of a theorem by Poincaré [1]. We report here a simplified version of such a theorem, concerned with plane foci.

Theorem 1 (Poincaré) Let (1) be analytic. Assume the origin to be a focus of (1). If the eigenvalues of (1) at $O$ have non-zero real part, then there exists a local analytic diffeomorphism at $O$ that linearizes (1).

If the eigenvalues at a focus have non-zero real part, the focus is said to be strong; otherwise, the focus is said to be weak. Theorem 1 shows that every strong focus of an analytic system has isochronous sections. In order to study cases not covered by theorem 1, in the following we shall be concerned with analytic weak foci, or with non-analytic foci.

In next theorem we consider generalized Liénard equations (4) where $f(x), g(x)$ are $C^{1}$ functions defined in a neighbourhood of the origin. Such equations are usually studied by means of the equivalent systems

$$
\dot{x}=y, \quad \dot{y}=-g(x)-y f(x),
$$

and

$$
\dot{x}=y-F(x), \quad \dot{y}=-g(x),
$$

where $F^{\prime}(x)=f(x), F(0)=0$. When studying isochronicity, it is more convenient to consider a third system equivalent to (4). Let us set

$$
I(x)=\int_{0}^{x} s f(s) d s
$$

Then the system we consider is

$$
\dot{x}=y-x B(x), \quad \dot{y}=-C(x)-y B(x),
$$

where

$$
B(x)=\left\{\begin{array}{cc}
I(x) / x^{2}, & x \neq 0 \\
f(0) / 2, & x=0
\end{array} \quad C(x)=\left\{\begin{array}{cc}
g(x)-x B(x)^{2}, & x \neq 0 \\
0, & x=0
\end{array}\right.\right.
$$

Remark 2 In [10], under the hypothesis $f(0)=0, g(0)=0$, it was proved that if $f$ and $g$ are $C^{1}$ functions, then also $B(x)$ and $C(x)$ are of class $C^{1}$. The same conclusion holds if $f(0) \neq 0$. In particular, $B(x)=\frac{f(0)}{2}+\frac{f^{\prime}(0) x}{3}+o\left(x^{2}\right), C(x)=g(x)-\frac{f(0)^{2} x}{4}+o(x)$.

In next theorem we assume the origin to be a stable focus, so that the solutions exist for all $t>0$. Similar statements can be proved if the origin is an unstable focus.

We denote by $y^{+}$the positive $y$ semi-axis $\{x=0, y>0\}$; by $y^{-}$the negative $y$ semi-axis $\{x=0, y<0\}$. We set $\epsilon=\sqrt{g^{\prime}(0)-\frac{f(0)^{2}}{4}}$. 
Theorem 2 Let $f, g \in C^{1}(\mathbb{R}, \mathbb{R}), g(0)=0, g^{\prime}(0)>0$. Let $O$ be a stable focus of (7). Assume that there exists $\nu>0$ such that $C(x)=\nu x$.

Then $\nu=g^{\prime}(0)-\frac{f(0)^{2}}{4}=\epsilon^{2}$, and

(i) the rays $Z_{\phi}=(\tau \cos \phi, \epsilon \tau \sin \phi), \tau>0$, are isochronous sections of (8);

(ii) the curves $L_{\phi}=(\tau \cos \phi, \epsilon \tau \sin \phi+F(\tau \cos \phi)-\tau \cos \phi B(\tau \cos \phi)), \tau>0$, are isochronous sections of (7);

(iii) the curves $S_{\phi}=(\tau \cos \phi, \epsilon \tau \sin \phi-\tau \cos \phi B(\tau \cos \phi)), \tau>0$, are isochronous sections of $(6)$.

A solution of (8) ((7), (6)), starting at a curve $Z_{\phi}$ (resp. $\left.L_{\phi}, S_{\phi}\right)$, at time $t$ crosses the curve $Z_{\phi-\epsilon t}$ (resp. $\left.L_{\phi-\epsilon t}, S_{\phi-\epsilon t}\right)$. In particular, $y^{+}$and $y^{-}$are isochronous sections of (6), (7) and (8). A complete revolution around the origin takes time $\frac{2 \pi}{\epsilon}$. A solution starting at a point of $y^{+}$crosses $y^{-}$after time $\frac{\pi}{\epsilon}$.

Proof.

We have $f(x)=f(0)+o(1)$, hence $x B(x)^{2}=\frac{f(0)^{2}}{4} x+o(x)$. If $C(x)=\nu x$, then $\nu x=g(x)-\frac{f(0)^{2}}{4} x+o(x)=g^{\prime}(0) x-\frac{f(0)^{2}}{4} x+o(x)$ that implies $\nu=g^{\prime}(0)-\frac{f(0)^{2}}{4}$.

(i) First we study isochronous sections of (8). By the hypothesis the system (8) appears as follows

$$
\dot{x}=y-x B(x), \quad \dot{y}=-C(x)-y B(x)=-\nu x-y B(x) .
$$

Performing the linear change of variables $u=x, v=\frac{y}{\epsilon}$, we get the system

$$
\dot{u}=\epsilon v-u B(u), \quad \dot{y}=-\epsilon u-v B(u),
$$

whose angular speed is $\dot{\theta}=-\epsilon$. Now we can apply lemma 1 , in order to prove that rays are isochronous sections. A solution starting at a point of the ray $\zeta_{\phi}$ of equations $u=\tau \cos \phi, v=\tau \sin \phi, \tau>0$, after time $t$ is on the ray $\zeta_{\phi-\epsilon t}$.

Applying the inverse transformation, $x=u, y=\epsilon v$, we prove that the rays $x=$ $\tau \cos \phi, y=\epsilon \tau \sin \phi, \tau>0$, are isochronous sections of (8). Moreover, a solution starting at a point of the ray $Z_{\phi}$, after time $t$ is on the ray $Z_{\phi-\epsilon t}$.

(ii) The transformation $(x, y) \mapsto(x, y+F(x)-x B(x))$ takes (8) into (7). The ray $Z_{\phi}$ is taken into the curve $L_{\phi} \equiv\{(x, y): x=\tau \cos \phi, y=\epsilon \tau \sin \phi+F(\tau \cos \phi)-$ $\tau \cos \phi B(\tau \cos \phi), \tau>0\}$. As in the previous point, a solution starting at a point of the curve $L_{\phi}$, after time $t$ is on the curve $L_{\phi-\epsilon t}$.

(iii) The transformation $(x, y) \mapsto(x, y-F(x))$ takes (7) into (6). The curve $L_{\phi}$ is taken into the curve $S_{\phi} \equiv\{(x, y): x=\tau \cos \phi, y=\epsilon \tau \sin \phi-\tau \cos \phi B(\tau \cos \phi), \tau>0\}$. As in point (i), a solution starting at a point of the curve $S_{\phi}$, after time $t$ is on the curve $S_{\phi-\epsilon t}$.

In all of these cases, choosing $\phi= \pm \frac{\pi}{2}$, one obtains the semi-axes $y^{+}$and $y^{-}$as isochronous sectios. Moreover, since the angular speed of (9) is $\dot{\theta}=-\epsilon$, a complete revolution around the origin is performed in time $\frac{2 \pi}{\epsilon}$. Passing from $y^{+}$to $y^{-}$requests half time, that is $\frac{\pi}{\epsilon}$.

For $\cos \phi \neq 0$, the curves $L_{\phi}$ are contained in the graphs of the functions $y=$ $\epsilon x \tan \phi+F(x)-x B(x)$. Similarly, for $\cos \phi \neq 0$, the curves $S_{\phi}$ are contained in the graphs of the functions $y=\epsilon x \tan \phi-x B(x)$. 
Theorem 2 has a simple physical interpretation. When the origin is a stable focus and $C(x)=\nu x$, the physical system modelled by (4) goes through infinitely many damped oscillations, touching the position $x=0$ at equal time intervals of width $\frac{\pi}{\sqrt{g^{\prime}(0)-\frac{f(0)^{2}}{4}}}$. An example of Liénard equation exhibiting such a behaviour is given by

$$
\ddot{x}+4 x^{2} \dot{x}+x+x^{5}=0 .
$$

Standard arguments show that the origin is a focus. Notice that the eigenvalues of the system at $\mathrm{O}$ are $\pm i$, hence theorem 1 does not apply.

Under the hypotheses of theorem 2, the systems (9), (8), (7), (6) have just one critical point, since, for system (9), one has $\dot{\theta}=-\epsilon$.

Assume (1) to be analytic. By applying Poincaré's theorem, one can prove the existence of a transformation $\Lambda$ taking (1) into a linear one.

$$
\dot{x}=a x+b y \quad \dot{y}=c x+d y,
$$

The system (11) commutes with

$$
\dot{x}=x, \quad \dot{y}=y,
$$

whose orbits are just rays. The inverse transformation $\Lambda^{-1}$ takes the orbits of (12) into orbits of a system commuting with (1). Such orbits are isochronous sections of (1).

It is natural to ask whether the orbits of a commutator are always isochronous sections of (1). If true, this would allow to avoid the restrictions related to the hypotheses of Poincaré's theorem, so considering also non-analytic systems. Also, Poincaré's theorem proves only the existence of a linearization, but does not show how to construct it. On the other hand, if a commutator was known, then its orbits would give the desired sections. Since several couples of commuting system possess symmetry properties (see [2]), often it would be possible to find the isochronous sections as symmetry axes.

Here we adapt the commutators' method, in order to find isochronous sections. We first examine the conditions for a couple of linear systems to be nontrivial commutators. Let us consider a second linear system

$$
\dot{x}=\alpha x+\beta y \quad \dot{y}=\gamma x+\delta y,
$$

Lemma 2 The linear systems (11) and (13) commute if and only if the matrix

$$
\left(\begin{array}{ccc}
a-d & b & c \\
\alpha-\delta & \beta & \gamma
\end{array}\right)
$$

has rank 1.

Proof. The commutativity conditions are

$$
\left\{\begin{array}{l}
(b \gamma-c \beta) x+(a \beta+b \delta-b \alpha-d \beta) y=0 \\
(c \alpha+d \gamma-a \gamma-c \delta) x+(c \beta-b \gamma) y=0
\end{array}\right.
$$

Equating to zero the coefficients of $x$ and $y$, one obtains the conditions

$$
\left|\begin{array}{ll}
b & c \\
\beta & \gamma
\end{array}\right|=\left|\begin{array}{cc}
b & a-d \\
\beta & \alpha-\delta
\end{array}\right|=\left|\begin{array}{cc}
c & a-d \\
\gamma & \alpha-\delta
\end{array}\right|=0
$$

which are equivalent to the condition of the statement. 
Lemma 3 Assume the linear systems (11) and (13) to commute. If $(a-d, b, c) \neq$ $(0,0,0)$, then they are transversal at noncritical points if and only if $(a-d)^{2}+4 b c<0$.

Proof. The transversality condition for (11) and (13) is

$$
(a \gamma-\alpha c) x^{2}+(a \delta+b \gamma-\alpha d-\beta c) x y+(b \delta-\beta d) y^{2} \neq 0, \quad \forall(x, y) \neq(0,0) .
$$

By the commutativity condition of the previous lemma, there exists $\mu \neq 0$ such that $(\alpha-\delta, \beta, \gamma)=\mu(a-d, b, c)$. Hence $\alpha=\mu(a-d)+\delta$. Substituting such a value into the transversality condition, we obtain

$$
c(\mu d-\delta) x^{2}+(\delta-\mu d)(a-d) x y+b(\delta-\mu d) y^{2} \neq 0, \quad \forall(x, y) \neq(0,0) .
$$

One cannot have $\delta-\mu d=0$, otherwise (11) and (13) would be parallel. Hence the above condition reduces to

$$
-c x^{2}+(a-d) x y+b y^{2} \neq 0, \quad \forall(x, y) \neq(0,0),
$$

which holds if and only if $(a-d)^{2}+4 b c<0$.

$$
\text { If }(a-d, b, c)=(0,0,0) \text {, then }(11) \text { is the radial system }
$$

$$
\dot{x}=a x \quad \dot{y}=a y,
$$

which commutes with every linear system. Elementary computations show that in this case the transversality condition of (17) and (13) reduces to $(\alpha-\delta)^{2}+4 \beta \gamma<0$, which is the condition of the above lemma, stated for system (13).

Lemma 4 If the linear systems (11) and (13) are nontrivial commutators, then $O$ is either a center, or a focus, or a star node of (11).

Proof. Assume $\mathrm{O}$ not to be a star node for $(11)$, so that $(a-d, b, c) \neq(0,0,0)$. The eigenvalues of (11) are

$$
\lambda_{1,2}=\frac{a+d \pm \sqrt{(a-d)^{2}+4 b c}}{2} .
$$

By the above lemma, one has $(a-d)^{2}+4 b c<0$, hence the eigenvalues have non-zero imaginary part, so that $\mathrm{O}$ can only be a center or a focus.

In next theorem we show that under suitable hypotheses the orbits of a commutator are isochronous sections of a given system.

Theorem 3 If the vector field $W$ has a focus $O$ and a nontrivial commutator $W_{1}$ with a star node at $O$, then every orbit of $W_{1}$ contained in a neighbourhood of $O$ is an isochronous section of $W$.

Proof. Since the origin is a focus for $W$, there exists a neighbourhood $U$ of $\mathrm{O}$ such that for every $W$-solution starting at a point $z \in U, \lim _{t \rightarrow+\infty} \theta(t, z)=+\infty$. On the other hand, possibly considering $-W_{1}$ instead of $W_{1}$, there exists a neighbourhood $U^{\prime}$ of $\mathrm{O}$ such that for every $W_{1}$-solution starting at a point $z \in U^{\prime}, \lim _{s \rightarrow+\infty} r(s, z)=0$. 
Without loss of generality we can assume $U=U^{\prime}$. All the $W$ - and $W_{1}$-solutions starting at points of $U=U^{\prime}$ exist for all positive times, hence the flows commute in $U=U^{\prime}$. Moreover, all the $W_{1}$-solutions tend to the origin having a limit tangent. Let us choose arbitrarily any of such solutions, $\eta$. Since $\eta$ has a limit tangent at O, every $W$-solution $\psi$ starting at a point $z$ of $\eta$ meets $\eta$ infinitely many times. Let $\eta(S, z)=\psi(T, z)$ be the first intersection of $\eta$ and $\psi$ after $z=\eta(0, z)=\psi(0, z)$. In other words, for all $\sigma \in(0, S)$, for all $\tau \in(0, T), \eta(\sigma, z) \neq \psi(\tau, z)$. Moreover, by the transversality of two vector fields, the arcs of solutions $\psi(t, z), t \in[0, T], \eta(s, z), s \in[0, S]$ bound a region which is positively invariant both for $W$ and for $W_{1}$. Hence $\psi(t, z)$ cannot meet the arc $\eta(s, z), s \in(0, S)$ for any value of $t \in \mathbb{R}$. Similarly, $\eta(s, z)$ cannot meet the arc $\psi(t, z), t \in(0, T)$ for any value of $s \in \mathbb{R}$.

(i) By the commutativity, one has

$$
\psi(2 T, z)=\psi(T, \eta(S, z))=\eta(S, \psi(T, z))=\eta(2 S, z) .
$$

Similarly, one proves that, for every positive integer $n$,

$$
\psi(n T, z)=\eta(n S, z) .
$$

Hence, property (i) of Definition 2 holds.

(ii) Assume by absurd that there exists $w=\eta(s, z)=\psi(t, z)$, with $t>0, t \neq n T$ for every positive integer $n$. Let $m$ be a positive integer such that $m T<t<(m+1) T$ There exists no positive integer $k$ such that $s=k S$, otherwise $\psi(t, z)=\eta(s, z)=$ $\eta(k S, z)=\psi(k T, z)$, with $t \neq k T$. Hence there exists a positive integer $k$ such that $k S<s<(k+1) S$. Let us set $\tau=t-m T, \sigma=s-k S$. As in point (i), one proves that for every integer $n$ such that both $n \tau \leq(m+1) T$ and $n \sigma \leq(k+1) S$, one has $\psi(-n \tau, w)=\eta(-n \sigma, w)$. Since $\tau<T, \sigma<S$, there exists a positive integer $h$ such that $0<h \tau<(m+1) T, 0<h \sigma<(k+1) S$ and either $0<h \tau<T$, or $0<h \sigma<S$. Then $\psi(-h \tau, w)=\eta(-h \sigma, w)$, that contradicts the fact that $\eta(s, z)$ cannot meet the arc $\psi(t, z), t \in(0, T)$ for any value of $s \in \mathbb{R}$.

Poincaré's theorem cannot be applied to non analytic systems. In some cases we can apply next corollary. In next corollary we show that every system with a focus and a nontrivial commutator has a commutator with a star node, hence, it has an isochronous section. As in [9], we say that $W_{\phi}, \phi \in[0,2 \pi)$, is a complete family of commuting vector fields if $W_{\phi}, \phi \in[0,2 \pi)$, is a complete family of rotated vector fields and for every couple $\phi, \phi^{\prime} \in[0,2 \pi)$, the vector fields $W_{\phi}, W_{\phi^{\prime}}$ commute.

Corollary 1 If the vector field $W$ has eigenvalues with non-zero real part at a focus $O$ and a nontrivial commutator $W_{1}$, then it has an isochronous section.

Proof. It is sufficient to show that $W$ has a nontrivial commutator with a star node at $\mathrm{O}$. Without loss of generality, we can assume $\mathrm{O}$ to be stable. By Theorem 1.4 in [9] the complete family $W_{\phi}=W \cos \phi+W_{1} \sin \phi, \phi \in[0,2 \pi)$, contains an isochronous center. Let $W_{2}$ be the corresponding vector field. Let us consider the second complete of commuting fields defined by $W_{\phi}^{*}=W \cos \phi+W_{2} \sin \phi, \phi \in[0,2 \pi)$. There exists a linear transformation $\Lambda$ taking $W_{2}$ into a vector field $V_{2}$ having linear part at $\mathrm{O}$ of the form

$$
\left(\begin{array}{cc}
0 & 1 \\
-1 & 0
\end{array}\right)
$$


The transformation $\Lambda$ takes the family $W_{\phi}^{*}$ into the complete family of commuting vector fields $V_{\phi}=V \cos \phi+V_{2} \sin \phi$, where $V$ is the vector field obtained from $W$ by means of $\Lambda$.

By Lemma 2, the linear part of $V$ at $O$ is of the form

$$
\left(\begin{array}{cc}
a & b \\
-b & a
\end{array}\right), \quad a \neq 0
$$

so that the linear part of $V_{\phi}$ at $\mathrm{O}$ is

$$
\left(\begin{array}{cc}
a \cos \phi & b \cos \phi+\sin \phi \\
-b \cos \phi-\sin \phi & a \cos \phi
\end{array}\right)
$$

Its eigenvalues are the solutions to the equation

$$
(\lambda-a \cos \phi)^{2}+(b \cos \phi+\sin \phi)^{2}=0 .
$$

For $\phi=\arctan (-b)$ the above matrix has the double real eigenvalue $\lambda=a \cos \phi$. The corresponding vector field $V_{\arctan (-b)}$ has a star node at $\mathrm{O}$. Let $W_{3}$ be the vector field obtained from $V_{\arctan (-b)}$ by means of the inverse transformation $\Lambda^{-1} . W_{3}$ is a nontrivial commutator of $W$, having a star node at $O$. Then we can apply Theorem 3 .

If the eigenvalues of $W$ at a focus $O$ have zero real part, then the complete family of the theorem does not necessarily contain a star node. In fact, the systems

$$
\dot{x}=y, \quad \dot{y}=-x,
$$

and

$$
\dot{x}=y+x\left(x^{2}+y^{2}\right), \quad \dot{y}=-x+y\left(x^{2}+y^{2}\right),
$$

commute, but the complete family they generate does not contain any singular point of node type. In this case it is possible to find a commutator of (22) whose orbits are rays,

$$
\dot{x}=x\left(x^{2}+y^{2}\right), \quad \dot{y}=y\left(x^{2}+y^{2}\right) .
$$

We cannot say that such a system has a star node at $\mathrm{O}$ because its eigenvalues at $\mathrm{O}$ are zero.

Using complete families it is easy to construct systems having foci with isochronous sections. In fact, assume $W$ to have an isochronous center at $\mathrm{O}$, and $W_{1}$ to be a nontrivial commutator such that $\mathrm{O}$ is a star node for $W_{1}$. Arguing as in 1 one shows that every vector field of the complete family $W_{\phi}=W \cos \phi+W_{1} \sin \phi, \phi \neq 0, \pi / 2, \pi, 3 \pi / 2$, has a focus at $\mathrm{O}$, and commutes with $W_{1}$. The orbits of $W_{1}$ are isochronous sections for the vector fields $W_{\phi}$. For example, we can take a couple of conjugate harmonic functions $P(x, y)=-y+p(x, y)$ and $Q(x, y)=x+q(x, y)$, vanishing at the origin. Taking $W \equiv(P, Q)$ and $W_{1} \equiv(-Q, P)$ we have a couple of commuting vector fields ([11], [2]). By theorem 1.3 in [8], $W$ has a center at $O$. Then every vector field of the family $W_{\phi}=W \cos \phi+W_{1} \sin \phi, \phi \neq 0, \pi / 2, \pi, 3 \pi / 2$, has a focus with isochronous sections, given by the orbits of $W_{1}$. Since usually such systems have symmetry axes, it is easy to find at least one isochronous section.

Linearizations of such systems are known, but not easy to use in order to find isochronous sections for the vector fields of the family $W_{\phi}$ (see [7], section 6). 
Several additional examples can be constructed from the systems listed in [2]. For some of such system, commutators are known, but linearizations have not yet been found.

\section{Isochronous sections of limit cycles}

Looking for isochronous oscillations of plane systems, we are led to consider also the behaviour of a system in the neighbourhood of a cycle. Since we do not consider only analytic systems, we can also have cycles $\Gamma$ bounding period annuli, orbitally stable on one side, attracting or repelling on the opposite side.

Definition 3 Let $\Gamma$ be a cycle of (1). Let $\eta:[0, \epsilon) \rightarrow \mathbb{R}^{2}$ be a $C^{1}$ curve such that $\eta(0) \in \Gamma, \eta(s) \notin \Gamma$ for $s>0$. Then we say that $\eta$ is a one-sided isochronous section of (1) at $\Gamma$ if either $\psi$ or $-\psi$ has the following property: there exists $T>0$ such that $\forall \psi(t, z)$ solution of (1), with $z \in \eta$, one has

(i) $\psi(n T, z) \in \eta$, for every positive integer $n$;

(ii) $\psi(t, z) \notin \eta$, for $t>0, t \neq n T$.

If $\eta:(-\epsilon, \epsilon) \rightarrow \mathbb{R}^{2}$ is a $C^{1}$ curve such that $\eta(0) \in \Gamma, \eta(s) \notin \Gamma$ for $s \neq 0$, we say that $\eta$ is an isochronous section of (1) at $\Gamma$ if both $\eta:[0, \epsilon) \rightarrow \mathbb{R}^{2}$ and $\chi(s)=\eta(-s)$ : $[0, \epsilon) \rightarrow \mathbb{R}^{2}$ are one-sided isochronous sections at $\Gamma$, lying on opposite sides of $\Gamma$.

From the definition it is evident that $T$ is $\Gamma$ 's minimal period. If $\Gamma$ has an isochronous section, then it has infinitely many isochronous sections, obtained from $\eta$ by means of the time maps $\psi(t, \cdot), 0<t<T$.

Remark 3 One-sided isochronous sections have been introduced in order to consider also cycles with different stability properties on opposite sides. For such cycles, neighbouring solutions could exist for all $t>0$ on one side of $\Gamma$, for all $t<0$ on the opposite side of $\Gamma$.

The orbital stability of limit cycles can be studied by means of its characteristic multipliers (see, e.g. [3]). If the characteristic multipliers of a limit cycle do not have modulus 1 , then the limit cycle is said to be hyperbolic. The existence of isochronous sections for hyperbolic limit cycles has been proved in [6]. Proving that a limit cycle is hyperbolic may be difficult, since limit cycles are often found by applying PoincaréBendixson theory, which gives a rough information about the localization of the limit cycle. Moreover, semi-stable cycles are not hyperbolic, so that some different technique is required in order to study isochronicity phenomena close to a semi-stable cycle.

Studying limit cycles we cannot consider linearizations, because linear systems do not have limit cycles. Also, commutators are not useful, since if a system has a nontrivial commutator, then it has no limit cycles (the argument of theorem 4.5 (b) in [11] extends to non-orthogonal commutators). The only argument of the previous section that can be applied to the study of isochronous sections of cycles is that of lemma 1 .

Lemma 5 Let $\Gamma$ be a limit cycle of (1). If there exists a diffeomorphism $\Lambda$ defined in a neighbourhood of $\Gamma$, taking (1) into a system having, in polar coordinates, the form

$$
\dot{\rho}=f(\rho, \theta), \quad \dot{\theta}=g(\theta)>0 .
$$

then (1) has an isochronous section at $\Gamma$. 
Proof. The proof is similar to that of lemma 1.

Modifying slightly the hypotheses of theorem 2, we can prove the existence of cycles with isochronous sections for a class of Liénard systems.

Let us set $G(x)=\int_{0}^{x} g(s) d s$. As in the proof of theorem 2 , we set $\epsilon=\sqrt{g^{\prime}(0)-\frac{f(0)^{2}}{4}}$.

Theorem 4 Let $f, g \in C^{1}(\mathbb{R}, \mathbb{R}), g(0)=0, g^{\prime}(0)>0$. Assume that

(1) there exists constants $c, \kappa_{1}>0$ such that $x F(x)>0$ for $|x|>\kappa_{1}$, and either $F(x)>c$ for $x>\kappa_{1}$, or $F(x)<-c$ for $x<-\kappa_{1}$;

(2) there exists $\kappa_{2}>0$ such that $x f(x)<0$ for $0<|x|<\kappa_{2}$;

(3) there exists $\nu>0$ such that $C(x)=\nu x$.

Then every cycle of (6), (7), (8) has two one-sided isochronous sections on opposite sides. Moreover, there exists at last a limit cycle with an isochronous section. The statements (i), (ii), (iii) of theorem (2) hold, considering $Z_{\phi}, L_{\phi}, S_{\phi}$ as isochronous sections at the limit cycle. In particular, $y^{+}$and $y^{-}$are isochronous sections of (6), (7) and (8). A complete revolution around the origin takes time $\frac{2 \pi}{\epsilon}$. A solution starting at a point of $y^{+}$crosses $y^{-}$after time $\frac{\pi}{\epsilon}$.

Proof. One can work as in theorem 2, proving that (8) can be transformed into a system with constant angular speed $\dot{\theta}=-\epsilon$. There exists a unique critical point, so that if a cycle exists, it surrounds the origin. Rays are isochronous sections of every cycle. Which kind of isochronous section depends on the stability properties of the cycle (see remark 3 ).

As for the existence of a cycle with a (bilateral) isochronous section, let us set $V(x, y)=y^{2}+2 G(x)$. Since $g^{\prime}(0)>0, V$ is positive definite at the origin. By assumption (2), the derivative of $V(x, y)$ along the solutions of (7) is $\dot{V}(x, y)=-2 g(x) F(x) \geq 0$ in a neighbourhood of $\mathrm{O}$, hence the origin is negatively asymptotically stable.

The hypotheses of theorem 3.1 in [5] hold. In fact, as in theorem 2, if $C(x)=$ $\nu x$, then $g(x)=\epsilon^{2} x+x B(x)^{2}$, so that $x g(x)=\epsilon^{2} x^{2}+x^{2} B(x)^{2}>0$ for all $x \neq 0$, and by the assumption (1) the inequalities (3.3)-(3.5) in [5] hold. As for the integral condition in the hypotheses of theorem 3.1 of [5], by condition (1) above we have $\int_{0}^{+\infty}(f(x)+|g(x)|) d x \geq \kappa_{3}+\int_{0}^{+\infty}|g(x)| d x$, where $\kappa_{3}$ is some constant. Moreover

$$
\int_{0}^{+\infty}|g(x)| d x=\int_{0}^{+\infty}\left(\epsilon^{2} x+x B(x)^{2}\right) d x \geq \epsilon^{2} \int_{0}^{+\infty} x d x=+\infty .
$$

Similarly, one can prove that $\int_{0}^{-\infty}(f(x)+|g(x)|) d x=-\infty$.

As a conclusion, the solutions of (6), (7), (8) are ultimately bounded. This, together with the uniqueness of the critical point and its negative stability, shows the existence of an attracting annulus $\mathrm{A}$, having two limit cycles as boundary. If $\mathrm{A}$ contains a period annulus, then every cycle contained in the interior of the period annulus has an isochronous section.

Hence, let us assume that A does not contain any period annulus. There exists a Poincaré's map $\Pi(y)$ (we identify the point $(y, 0)$ with the number $y$; as well as $(\Pi(y), 0)$ and $\Pi(y))$, defined in a neighbourhood of $A \cap y^{+} \equiv\left[y_{1}, y_{2}\right]$. Either the fixed points of $\Pi$ are finitely many, or they accumulate at a point. 
If they are finitely many, then the map $\Pi(y)-y$ has finitely many zeroes. Since A is attracting, $\Pi(y)-y>0$ for $y<y_{1}, \Pi(y)-y<0$ for $y>y_{2}$, close enough to $\left[y_{1}, y_{2}\right]$. Hence $\Pi(y)-y$ has to change sign, from positive to negative, at least at a point $y_{*}$. The orbit through $\left(0, y_{*}\right)$ is an asymptotically stable cycle, for which $y^{+}$is an isochronous section.

If they are infinitely many, they accumulate at a point $\left(0, y_{*}\right)$. Since they all have the same period, by continuity the orbit passing through $P_{*} \equiv\left(0, y_{*}\right)$ is a cycle $\Gamma_{*}$. Elementary stability arguments show that $\Gamma_{*}$ is stable. Also in this case, $y^{+}$is an isochronous section.

Corollary 2 Let $f, g$ be analytic, $g(0)=0, g^{\prime}(0)>0$. Assume that the hypotheses of theorem (4) hold. Then there exists an asymptotically stable limit cycle with an isochronous section.

Proof. If $f$ and $g$ are analytic, then Poincaré's map is analytic, hence its fixed points cannot accumulate. This implies that A contains finitely many cycles. Working as in the previous proof, one shows that one of them is asymptotically stable.

By choosing $f(x)=6 x^{4}-4 x^{2}$ we get an example of Liénard system with a limit cycle having $y^{+}$and $y^{-}$as an isochronous sections,

$$
\dot{x}=y, \quad \dot{y}=\left(6 x^{4}-4 x^{2}\right) y-x-x^{5}+2 x^{7}-x^{9} .
$$

Such a system is equivalent to the equation

$$
\ddot{x}+\left(6 x^{4}-4 x^{2}\right) \dot{x}+x+x^{5}-2 x^{7}+x^{9}=0,
$$

whose solutions assume the position $x=0$ at equal time intervals.

\section{Acknowledgements}

The author would like to thank prof. J. Chavarriga and J. Giné of the Univ. of Lleida for several useful conversations.

\section{References}

[1] V. I. ARnold, Geometrical methods in the theory of ordinary differential equations, New York, 1983.

[2] J. Chavarriga, M. Sabatini A survey of isochronous centers, Qual. Theory Dyn. Syst. 1 (1999), no. 1, 1-70.

[3] C. Chicone, Ordinary differential equations with applications, Springer Verlag, Berlin, 1999.

[4] R. Conti Uniformly isochronous centers of polynomial systems in $\mathbb{R}^{2}$, Lecture Notes in Pure Appl; Math., vol. 152, pp. 21-31, M. Dekker, New York, 1994. Qual. Theory Dyn. Syst. 1 (1999), no. 1, 71-95.

[5] J. R. Graef On the generalized Liénard equation with negative damping, J. Differential Equations 12 (1972), 34-62. 
[6] J. GuCKenheimer Isochrons and phaseless sets, J. Math. Biology 1 (1975), 259273.

[7] P. Mardes̆ić, C. Rousseau and B. Toni, Linearization of isochronous centers, J. Differential Equations 121 (1995), 67-108.

[8] L. Mazzi, M. Sabatini Commutators and linearizations of isochronous centers, Rend. Mat. Acc. Lincei, s. 9, 11 (2000), 81-98.

[9] M. Sabatini Dynamics of commuting systems on two-dimensional manifolds, Ann. Mat. Pura Appl.(4) 173 (1997), 213-232.

[10] M. Sabatini On the period function of Liénard systems, J. Differential Equations 152 (1999), no. 2, 467-487.

[11] M. VILLARINI Regularity properties of the period function near a center of a planar vector field,Nonlinear Anal., T. M. A. 19 (1992), no. 8, 787-803. 